\title{
Complete Resolution of Cerebral Air Embolism Secondary to a Transbronchial Needle Aspiration
}

\author{
S. Perinel Ragey ${ }^{\mathrm{a}} \quad$ P. Garnier ${ }^{\mathrm{b}} \quad$ J.-M. Vergnon ${ }^{\mathrm{a}}$
}

${ }^{a}$ Department of Chest Diseases and Thoracic Oncology, University Hospital of St. Etienne and LINA, Jean Monnet University, and ${ }^{b}$ Stroke Unit, Department of Neurology, University Hospital of St. Etienne, St. Etienne, France

\section{Established Facts}

- Air embolism following transbronchial needle aspiration (TBNA) is extremely rare but is often fatal.

- The treatment of these events involves the use of oxygen and hyperbaric therapy, but evidence of efficacy is lacking.

\section{Novel Insights}

- To our knowledge, this is the first reported case of a successful therapy for cerebral air embolism following TBNA.

- Our study shows the importance, for each practitioner of TBNA, of being aware of this side effect and its early treatment despite its rarity.

\section{Key Words}

Transbronchial needle aspiration · Bronchoscopy · Cerebral air embolism · Epilepsy · Hyperbaric therapy

\footnotetext{
Abstract

Air embolism following transbronchial needle aspiration (TBNA) is extremely rare. To date, only 1 case has been described (estimated incidence: 0.02-0.07\%). Here, a 70-yearold patient developed a left upper-lobe alveolar syndrome with no response to well-conducted antibiotic treatments. Conventional bronchoscopy was normal, but virtual bronchoscopy showed that the tumor was contiguous to the left B3-B4 division. During a second bronchoscopy, a TBNA was performed without bleeding, and pathology later revealed
}

primitive lung adenocarcinoma. Immediately after the puncture, an epileptic seizure occurred with right rotation of the head and a tonic seizure of the left arm followed by left hemiplegia. A brain CT scan performed almost immediately showed no abnormalities and a chest radiography did not show any pleural or mediastinal aeric image. A few hours later, clonic seizures occurred and brain MRI showed lesions compatible with air embolisms (right gyral cortical location). Several hyperbaric therapy sessions successfully cured the neurological deficit. Despite its rarity, each practitioner of TBNA has to be aware of this side effect and its early treatment. The mechanism of such accidents remains unclear, but probably involves high bronchial pressure and venous trauma.

(c) 2013 S. Karger AG, Basel

\section{KARGER}

E-Mail karger@karger.com www.karger.com/res
(C) 2013 S. Karger AG, Basel

0025-7931/13/0866-0504\$38.00/0
Prof. J.M. Vergnon

Department of Chest Diseases and Thoracic Oncology

Hôpital Nord, University Hospitals of St. Etienne

FR-42055 St. Etienne Cedex 2 (France)

E-Mail jean.michel.vergnon@ univ-st-etienne.fr 


\section{Introduction}

Flexible bronchoscopy is widely used to obtain histological or cytological samples as it is a safe procedure with an extremely low rate of complications [1] that can be performed under local anesthesia. Since 1985, transbronchial needle aspiration (TBNA) has significantly increased the sensitivity of this procedure $[2,3]$. TBNA is principally used to stage mediastinal nodes in lung cancer and to obtain a cytological diagnosis in peripheral pulmonary lesions contiguous to the airways [3].

Air embolisms following transbronchial biopsy or percutaneous CT scan-guided trans-thoracic needle aspiration are extremely rare but potentially fatal, with an estimated incidence of $0.02-0.07 \%$ [4]. These embolisms are even more unusual following TBNA. To our best knowledge, only 1 case has previously been described in the literature [5].

\section{Case Report}

The patient was a 70-year-old man suffering from multiple chronic diseases: diabetes mellitus with insulin therapy for 35 years (and complicated by peripheral neuropathy), severe COPD (FEV 1 approx. 35\%), age-related macular degeneration, arterial hypertension and dyslipidemia. He had been a smoker (30 pack-years), though he had quit 20 years earlier. He had worked as a mechanic all his life, but had experienced no dangerous exposure or suffered from any allergies.

He developed a left upper lobe alveolar syndrome with aeric bronchogram. His general practitioner first prescribed cefuroxime for 10 days, followed 1 month later by a second antibiotic therapy with amoxicillin-clavulanic acid in response to the progression of the alveolar syndrome on a control chest X-ray. In parallel, the worsening of dyspnea was treated by aerosols (terbutalin and ipratropium) and then oral corticosteroids. Two days after the beginning of the second course of antibiotic therapy, the worsening dyspnea led to hospitalization in an emergency unit. In the hospital, the patient presented no signs of infection (i.e. fever or inflammatory syndrome), but a PET scan showed intense FDG uptake in the pathological area (SUV max. 10.4). Moreover, a mediastinal node appeared in the aortopulmonary window (SUV max. 3.5). The conventional flexible bronchoscopy performed was macroscopically normal and all bacteriological, cytological and histological samples were negative (only squamous metaplasia).

Virtual bronchoscopy based on a CT scan showed that the suspected alveolar condensation was contiguous to the left B3-B4 division (fig. 1). Based on these data, a second bronchoscopy was carried out, under local anesthesia and without any sedation. It was used to perform additional bronchial biopsies, cytological brushing in B4-B5 bronchus and a TBNA. A 15-mm 19-G needle (Excelon, Boston, Mass., USA) was used to go through the carina and reach the target zone. A first pass was performed without noticeable bleeding (fig. 2). Other planned passes could not be carried out because, a few seconds later, the patient had a seizure with a right ro-

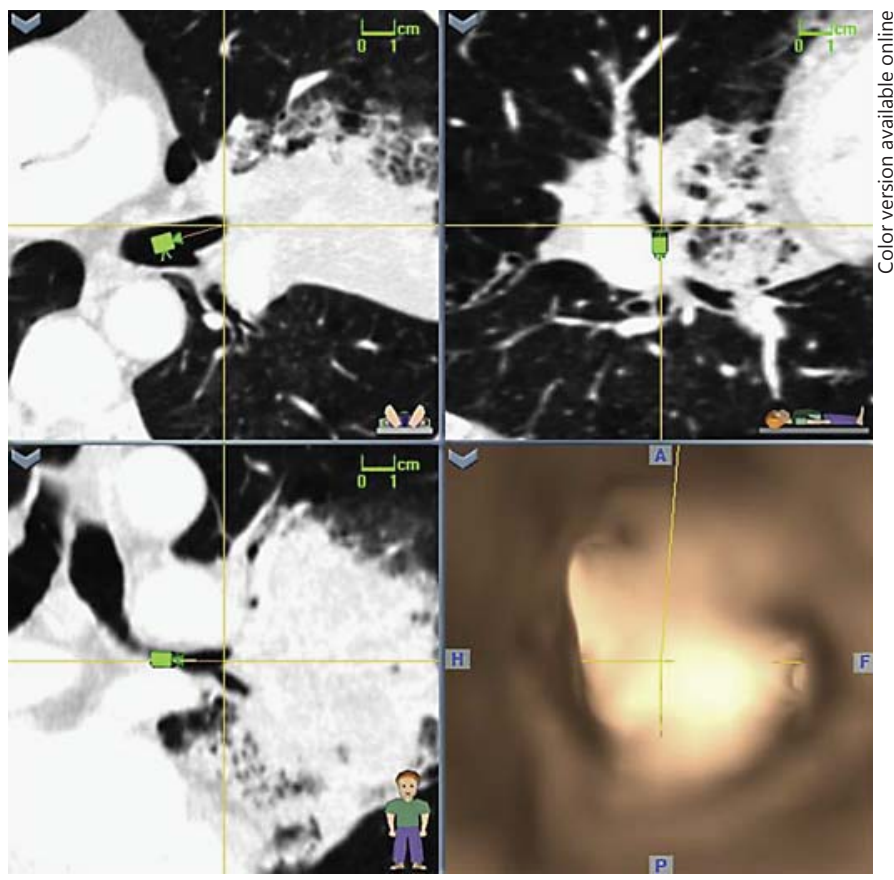

Fig. 1. Virtual bronchoscopy (integration 3D images of millimetric TDM). On the first 3 TDM images: the camera shows the axis of vision directly in front of the tumor, at the left $\mathrm{B} 3-\mathrm{B} 4$ division. The little man in the bottom right-hand corner shows the patient's position with respect to the camera. The virtual bronchoscopy is the last image down on the right. Both visually and virtually, no endobronchial abnormalities could be seen.

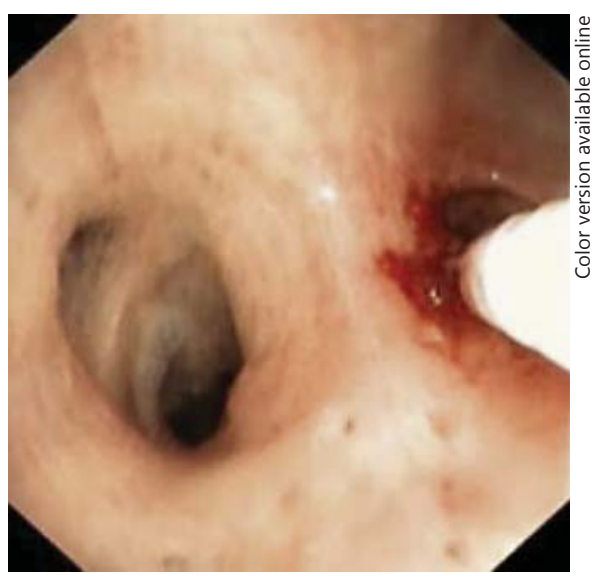

Fig. 2. The single TBNA, during the second bronchoscopy. Bleeding is minor. No other transbronchial punction was performed during the procedure. Cytology analysis would later reveal this to be a primitive adenocarcinoma. 
tation of the head and a tonic seizure of the left arm followed by left hemiplegia. A brain CT scan without injection performed almost immediately showed no abnormalities (air or other). Chest radiography failed to reveal any pleural or mediastinal air image and the supra-aortic Doppler was normal. The needle was in the correct position and inside the target; indeed, the cytological analysis of the needle sample revealed only lung adenocarcinoma.

The patient was immediately transferred to a neurological intensive care unit. On neurological examination, impaired consciousness and left hemiplegia with right rotation of the head were found. A few hours later, left recurrent focal motor seizures reappeared with hallucinations and impaired vigilance. The treatment consisted of clonazepam followed by phenytoin, but the patient's clinical status subsequently worsened with generalized tonic-clonic seizures and severely disturbed consciousness. A further CT scan without injection was unchanged and did not show any bleeding or ischemic lesion. The treatment was changed to clobazam and levetiracetam. Within the next few hours, his consciousness grade improved and the left hemiplegia partially regressed (essentially for the lower limb). Despite another normal brain CT scan without injection, brain MRI on sequences fluid-attenuated inversion recovery and diffusion-weighted images (DWI) showed findings compatible with air embolisms: right cortical gyral hyperintense lesions predominantly located in the frontoparietal border zone (fig. 3). Angio-MRI was normal. The treatment consisted of multi-session hyperbaric therapy, starting about $15 \mathrm{~h}$ after the initial symptoms. This successfully and totally cured the neurological deficit.

Following this event, the lung cancer was treated with two different chemotherapies, but the disease progressed and the patient died 6 months later without showing signs at any time of neurological deficit or cerebral metastases.

\section{Discussion}

TBNA was clearly indicated in this case where the evolution of the disease strongly pointed to lung cancer in the absence of a conclusive diagnosis from less invasive exams. TBNA has been widely used for a very long time (since 1985) and has a very low rate of complications $[2$, 3]. Sensitivity for peripheral nodules is $>40 \%$, increasing to $>60 \%$ for masses $>3 \mathrm{~cm}$, and higher for cancers. Medford et al. [6], according to a previous systematic review, found an overall diagnosis sensitivity of $73 \%$ for TBNA. Moreover, as Trisolini et al. [7] stated, TBNA is sensitive and cost-saving when used during routine bronchoscopy with diagnosis and staging purposes, especially when the pretest probability of pathological involvement is high. The use of TBNA under guidance increases the overall yield of bronchoscopy from 35 to $51 \%$ [2]. Considering the high probability of malignancy for this patient, this exam thus seemed justified.

In this case, TBNA was associated with a cerebral air embolism. There was ample evidence supporting this diagnosis: a predominant epileptic semiology as seen in air

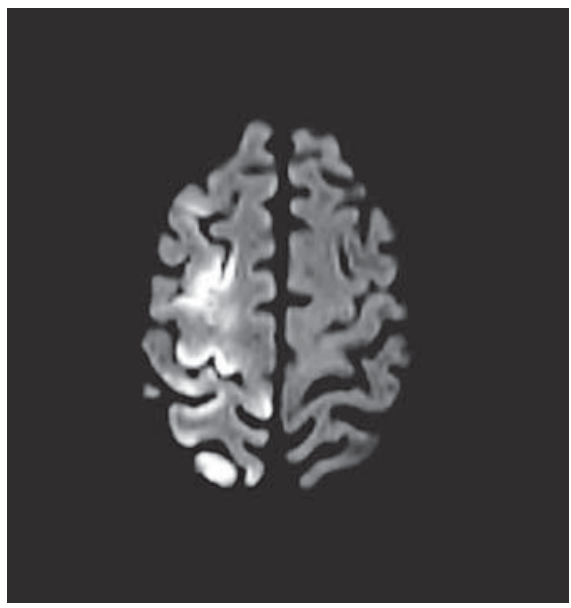

Fig. 3. Brain MRI on DWI sequence: lesions compatible with air embolisms, e.g. the right gyral cortical hyperintense lesion in frontoparietal border zone.

embolisms without response to benzodiazepine treatments, cortical and right distribution of lesions, especially in the cortical border zone, and the absence of lesions on every CT scan [8-11]. Moreover, no other diagnosis (such as ischemic lesion, blood pressure loss or hypoglycemia) appeared likely. There were also no signs of other complications of TBNA such as hemoptysis, pneumothorax, bronchospasm or pain. The subsequent development was also indicative of cerebral air embolism: clinical symptoms completely disappeared after hyperbaric treatment and no cerebral metastases or recurrence of this episode occurred.

Cerebral air embolisms are rare accidents that are poorly documented in the literature. A study in 2010 reported 2 fatal cases after bronchoscopic procedures [5]. However, 1 of these followed both TBNA and transbronchial biopsy and so it was not certain that it was a consequence of TBNA; despite oxygen therapy, the patient died a few hours later. The other case followed TBNA and hyperbaric oxygen therapy was initiated within $3 \mathrm{~h}$ of the bronchoscopy. After three sessions of hyperbaric treatment, only minor neurological recovery occurred. Importantly, these bronchoscopies were conducted under intravenous sedation (i.e. the delay in symptom onset and diagnosis is unknown). Another interesting article [4] documents the retrospective analysis of CT scans performed before and just after trans-thoracic needle biopsy. The results show that 3 of the 4 patients with air embolisms were totally asymptomatic, which suggests that the incidence of such pathologies is underevaluated. 
The mechanism of such accidents remains unclear, but probably involves high bronchial pressure (coughing is very common during the procedure) and venous trauma caused by the needle. Some authors speculate about a bronchovenous fistula or an immediate problem of coagulation and venoconstriction following the TBNA, associated with the cough. In this situation, air from the bronchi or alveoli could pass into the pulmonary veins, where a lower pressure could be observed at the inspiration phase.

In the case in our study, the procedure was performed under local anesthesia, without any sedation. This could be an important drawback for coughing and high pressure while TBNA is carried out, but this is the routine procedure in the unit because it avoids the risks of anesthesia and allows early observation of neurological symptoms or other complications.

The treatment of such accidents is based on oxygen and hyperbaric therapy [10]. These accidents are so rare, however, that no study has proven the efficacy of these therapies for this specific indication. The total cure of this event after hyperbaric therapy provides the first empirical evidence.

This case suggests that this severe side effect of TBNA can be reversed. Successful treatment of such accidents is strongly dependent upon early recognition of this event. Despite its rarity, we think that each practitioner performing TBNA should be aware of this event and its therapy.

\section{Acknowledgments}

The authors would like to thank Kirsty Burnet, $\mathrm{PhD}$, and Remi Vergnon, $\mathrm{PhD}$, for reviewing the manuscript.

\section{Financial Disclosure and Conflicts of Interest}

No conflicts of interest were declared by the authors.

\section{References}

$\checkmark 1$ Pue CA, Pacht ER: Complications of fiberoptic bronchoscopy at a university hospital. Chest 1995; 107:430-432.

-2 Reichenberger F, Weber J, Tamm M, Bolliger CT, Dalquen P, Perruchoud AP, et al: The value of transbronchial needle aspiration in the diagnosis of peripheral pulmonary lesions. Chest 1999;116:704-708.

-3 Gasparini S, Ferretti M, Secchi EB, Baldelli S, Zuccatosta L, Gusella P: Integration of transbronchial and percutaneous approach in the diagnosis of peripheral pulmonary nodules or masses. Experience with 1,027 consecutive cases. Chest 1995;108:131-137.
-4 Hiraki T, Fujiwara H, Sakurai J, Iguchi T, Gobara H, Tajiri N, et al: Nonfatal systemic air embolism complicating percutaneous CTguided transthoracic needle biopsy: four cases from a single institution. Chest 2007;132: 684-690.

5 Azzola A, Von Garnier C, Chhajed PN, Schirp U, Tamm M: Fatal cerebral air embolism following uneventful flexible bronchoscopy. Respiration 2010;80:569-572.

-6 Medford ARL, Agrawal S, Free CM, Bennett JA: A prospective study of conventional transbronchial needle aspiration: performance and cost utility. Respiration 2010;79: 482-489.

7 Trisolini R, Patelli M, Gasparini S: While waiting to buy a Ferrari, do not leave your current car in the garage! Respiration 2010;79: 452-453.
8 Hodics T, Linfante I: Cerebral air embolism. Neurology 2003;60:112.

9 Griese H, Seifert D, Koerfer R: Cortical infarction following cardiosurgical procedures - air embolism as a probable cause. Eur Neurol 2009;61:343-349.

10 Muth CM, Shank ES: Gas embolism. N Engl J Med 2000;342:476-482.

-11 Caulfield AF, Lansberg MG, Marks MP, Albers GW, Wijman CAC: MRI characteristics of cerebral air embolism from a venous source. Neurology 2006;66:945-946. 